\title{
Near-magnetic-field scaling for verification of spacecraft equipment
}

\author{
M. A. Pudney ${ }^{1}$, C. M. Carr ${ }^{1}$, S. J. Schwartz ${ }^{1}$, and S. I. Howarth ${ }^{2}$ \\ ${ }^{1}$ The Blackett Laboratory, Imperial College London, SW7 2AZ, UK \\ ${ }^{2}$ Astrium Ltd., Stevenage, SG1 2AS, UK \\ Correspondence to: M. A. Pudney (maxsim.pudney06@imperial.ac.uk)
}

Received: 16 May 2013 - Published in Geosci. Instrum. Method. Data Syst. Discuss.: 26 July 2013

Revised: 23 October 2013 - Accepted: 27 October 2013 - Published: 14 November 2013

\begin{abstract}
Magnetic-field measurements are essential to the success of many scientific space missions. Outside of the earth's magnetic field the biggest potential source of magnetic-field contamination of these measurements is emitted by the spacecraft. Spacecraft magnetic cleanliness is enforced through the application of strict ground verification requirements for spacecraft equipment and instruments. Due to increasingly strict $\mathrm{AC}$ magnetic-field requirements, many spacecraft units cannot be verified on the ground using existing techniques. These measurements must instead be taken close to the equipment under test (EUT) and then extrapolated. A traditional dipole power law of -3 (with a field fall-off proportional to $r^{-3}$ ) cannot be applied at these close distances without risk of underestimating the field emitted by the EUT, but we demonstrate that a power law of -2 is too conservative. We propose a compromise that uses a power law of -2 up to a distance equal to 3 times the unit size, beyond which a dipole power law can be applied. When extrapolating from a distance of $0.20 \mathrm{~m}$ to $1.00 \mathrm{~m}$ from the centre of a $0.20 \mathrm{~m}$ wide EUT, we demonstrate that this method avoids an under prediction of the field, and is at least twice as accurate as performing the extrapolation with a fixed power law of -2 .
\end{abstract}

\section{Introduction}

New space missions, such as the European Space Agency (ESA) Solar Orbiter mission (Müller et al., 2013), will make in situ measurements of the AC magnetic field that demand ever higher precision and accuracy. This is driven by search coil magnetometer (SCM) science requirements to determine the properties of kinetic particle interactions with the magnetic field in the solar wind, as well as other inner helio- spheric phenomena. These scientific requirements in turn drive strict ground verification requirements for spacecraft emitted AC magnetic fields, which are intended to ensure the spacecraft field stays sufficiently low (i.e. magnetic cleanliness is required). The overall spacecraft magnetic budget is broken down into individual unit requirements, including spacecraft equipment and scientific instruments. Before flight, ground magnetic testing is performed at unit level (individual items of equipments) and at spacecraft system level (all the units placed in their correct relative locations). We will focus on unit level testing, since the results from these tests give an earlier indication of potential contamination problems. We will also focus on AC magnetic-field frequencies below $1 \mathrm{MHz}$, in the operating frequency range of search-coil sensors for space science. At these frequencies the emissions can be treated as quasi-static, and therefore the same scaling rules that apply to DC magnetic fields can also be applied to AC fields.

A unit AC magnetic-field verification program of the rigour required by missions such as the Solar Orbiter demands deviation from standard test practices. Traditionally, an EUT has its magnetic-field characterised as a "black box" at a given distance (i.e. no assumption is made about the internal structure or electrical wiring before the field is measured). The field measurement is then extrapolated to the location of the SCM to assess whether the emission is acceptable and therefore meets the cleanliness requirements. It is important not to under predict or severely over predict the magnetic field emitted by the EUT. In order to perform this extrapolation, the field is traditionally assumed to vary with distance according to a power law of -3 from the centre of the EUT to the SCM. This is because the source of the field is assumed to be a simple dipole or current loop, with a field that follows a power law of -3 with distance (i.e. a field 


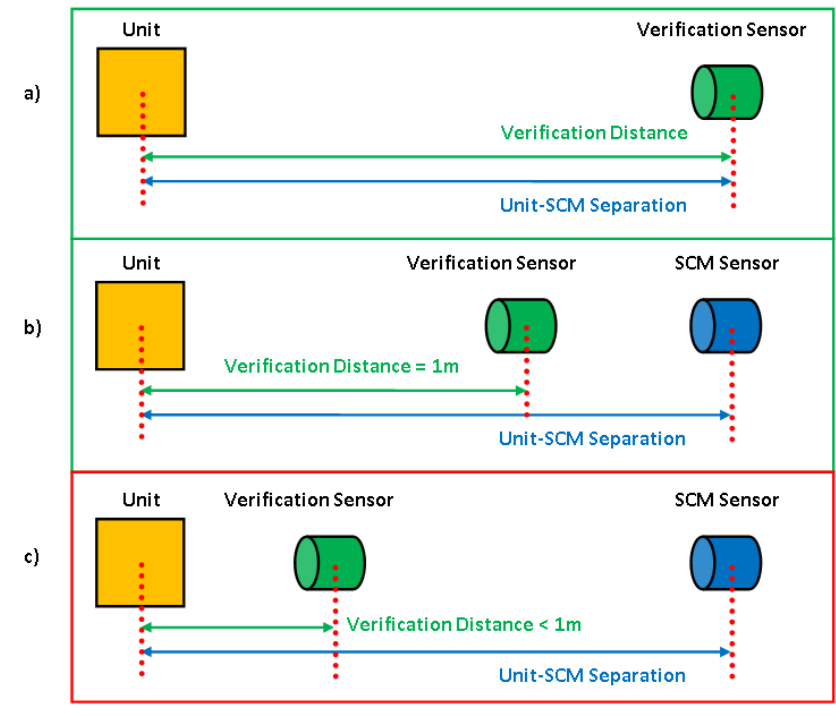

Fig. 1. Defining test distances (a) when the verification distance is the same as the unit-SCM separation distance, (b) when the verification distance is fixed at $1 \mathrm{~m}$, (c) when the verification distance must be closer than $1 \mathrm{~m}$.

fall-off that is proportional to $r^{-3}$ ) (Jackson, 1999). We assess whether this assumption is true and if this extrapolation can be improved.

During traditional unit AC field testing, the verification sensor is placed either at a distance equal to the unit-SCM separation on the spacecraft (Fig. 1a) or $1 \mathrm{~m}$ away from the EUT (Fig. 1b). However, difficulties arise when the verification field requirements are smaller than the noise floor of the magnetic test chamber. Under this condition the verification sensor must be placed closer than the unit-SCM separation and the field extrapolated over the remaining distance to obtain a predicted field emission (Fig. 1c). We define the distance between the EUT and verification sensor as the verification distance. This is the distance at which the field emission from the EUT will be measured during a test campaign.

In order to verify whether or not a unit magnetic-field emission passes or fails a mission's requirements, the following equation can be used:

$B_{\text {unit }}<B_{\text {req }}\left(\frac{d_{\text {ver }}}{d_{\text {sep }}}\right)^{n}$,

where $d_{\text {ver }}$ is the verification distance, $d_{\text {sep }}$ the in-flight unit to SCM separation (which we will refer to as the extrapolation distance), $B_{\text {req }}$ the science verification requirement and $n$ is the scaling power (traditionally -3 ). This equation can be adapted to find the maximum verification distance necessary to meet the requirements in a given test environment, yielding the equation:

$d_{\text {ver }}<d_{\text {sep }}\left(\frac{B_{\text {noise }}}{B_{\text {req }}}\right)^{\frac{1}{n}}$ where $B_{\text {unit }}$ has been replaced by $B_{\text {noise }}$, the level equivalent to the test chamber noise floor. It is assumed that the noise floor is measured with the same bandwidth as the verification requirement is specified (e.g. a narrow bandwidth of $1 \mathrm{~Hz}$ ).

If measurements must be made at close proximity to the EUT, a simple dipolar approximation with a scaling power of -3 cannot be assumed. Firstly, even an ideal centred current loop (with a diameter the same size as the unit) will generate a magnetic field that falls off more gradually than a power of -3 close to its centre. Secondly, since the location of dipoles within the EUT are not known a priori, they could be located anywhere within the volume of the unit. This means that there is a risk of underestimating the field and potentially accepting a unit that emits a larger field than the one extrapolated. While using a scaling power of -2 results in a safer field extrapolation, there is risk of significantly overestimating the field, such that the EUT fails the test unnecessarily.

Different scaling powers can be applied to different current geometries. For example, a simple single long thin wire will generate a field that falls off with a scaling power of -1 , with the equation (from Ampere's law):

$B=\frac{\mu_{0} I}{2 \pi r}$,

For comparison, in the context of transmission cables, a single circuit power line will follow a scaling power of -2 (Filippopoulos and Tsanakas, 2005), while a double circuit power line with transposed phasing with follow a scaling power of -3 (National Grid, 2012). On a smaller scale, at close distances, a current loop will follow a scaling power of -2 , while far from the loop the field follows a scaling power of -3 (Jackson, 1999).

We propose a compromise method that changes the scaling power from -2 to -3 after a predetermined distance has been reached, which we will from now on define as the break distance (as it is the break point between the use of the two scaling powers). Explicitly, we use a scaling power of -2 between the verification distance and break distance, then apply a scaling power of -3 between the break distance and the extrapolation distance. Beyond the break distance the dipole approximation is considered to be sufficiently valid for further extrapolation. Since magnetic measurements of the AC magnetic field using a narrow bandwidth (typically $1 \mathrm{~Hz}$ ) over a wide range of frequencies can take a long time, this work focusses on improving the extrapolation of the field from a single measurement. We model the magnetic-field emissions due to a series of small dipoles inside of a box.

First we discuss the finite element analysis model for calculating the magnetic field due to the flow of current. Since we only study AC fields, we do not include any magneticfield contributions due to the DC magnetisation of the unit material. We discuss the choice of current source inside the box, then investigate the impact of varying the verification distance and the break distance, comparing our method to 
Table 1. Centred single dipole model parameters.

\begin{tabular}{ll}
\hline Parameter & \\
\hline Loop radius & $0.10 \mathrm{~m}$ \\
Loop current & $1 \mathrm{~mA}$ \\
Loop position & Box centre \\
Loop orientation & on-axis $x$ axis \\
Number of loops & 1 \\
Box width & $0.20 \mathrm{~m}$ \\
Verification distance & $0.30 \mathrm{~m}$ \\
Break distance & $0.50 \mathrm{~m}$ \\
Extrapolation distance & $1.00 \mathrm{~m}$ \\
\hline
\end{tabular}

heritage techniques that use a fixed scaling power of -2 or -3 .

\section{Model procedure}

We use a finite element model (FEM) as described below to calculate the magnetic field of a chosen current geometry at any distance using MATLAB software. The Biot-Savart Law gives the general expression for the field contribution from an infinitesimal current element at any given point as

$\boldsymbol{d} \boldsymbol{B}=\frac{\mu_{0}}{4 \pi} \frac{I \boldsymbol{d} \boldsymbol{l} \times \boldsymbol{r}}{|\boldsymbol{r}|^{3}}$,

where $\boldsymbol{r}$ is the displacement vector from the current element to the point at which the field is being computed, and $\boldsymbol{d} \boldsymbol{l}$ the vector of the length element along the path of the current. The general expression for the total field is given by the integral over a closed path $c$ :

$\boldsymbol{B}=\oint_{c} \boldsymbol{d} \boldsymbol{B}$

where $\boldsymbol{d} \boldsymbol{B}$ is the infinitesimal field contribution from an element. By working with a FEM, we replace $\boldsymbol{d} \boldsymbol{l}$ with a finite current element vector $\delta \boldsymbol{l}$ such that the finite field contribution $\boldsymbol{\delta} \boldsymbol{B}$ from any individual element $l$ is given by

$\boldsymbol{\delta} \boldsymbol{B}=\frac{\mu_{0}}{4 \pi} \frac{I \delta \boldsymbol{l} \times \boldsymbol{r}}{|\boldsymbol{r}|^{3}}$

and the total magnetic field at any point is calculated by the sum of these field contributions:

$\boldsymbol{B}=\sum_{l=1}^{m} \boldsymbol{\delta} \boldsymbol{B}_{l}$

where $m$ is the total number of elements.

The magnetic field is calculated and analysed by using the following procedure:

1. A box size is selected, marking the boundary of the current loop locations.
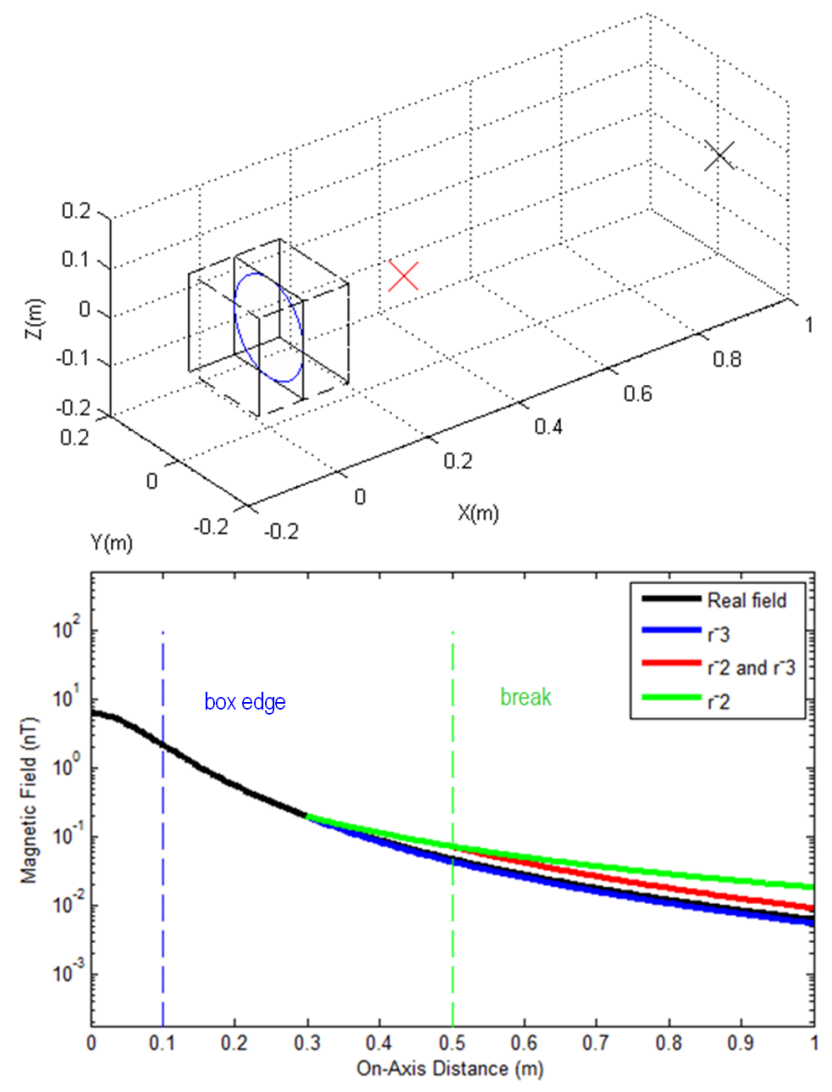

Fig. 2. (a) Model orientation set-up for a single centred dipole. The red cross indicates the measurement location and the black cross indicates the extrapolation location. (b) Modelling the field fall-off from a centred single dipole.

2. The number of current loops, radius, current, position and orientation are chosen depending on the test.

3. A verification position is chosen and a verification "measurement" made by calculating the magneticfield vector at the verification position using the BiotSavart Law.

4. The magnetic-field magnitude at the verification position is then used to extrapolate out to the extrapolation distance, using either

a. a scaling power of -2 from the verification distance to the extrapolation distance;

b. a scaling power of -3 from the verification distance to the extrapolation distance; or

c. a scaling power of -2 from the verification distance to a pre-selected break distance, then a scaling power of -3 from the break distance to the extrapolation distance.

Since in reality we would not generally know the geometry inside the box, this extrapolation is performed 

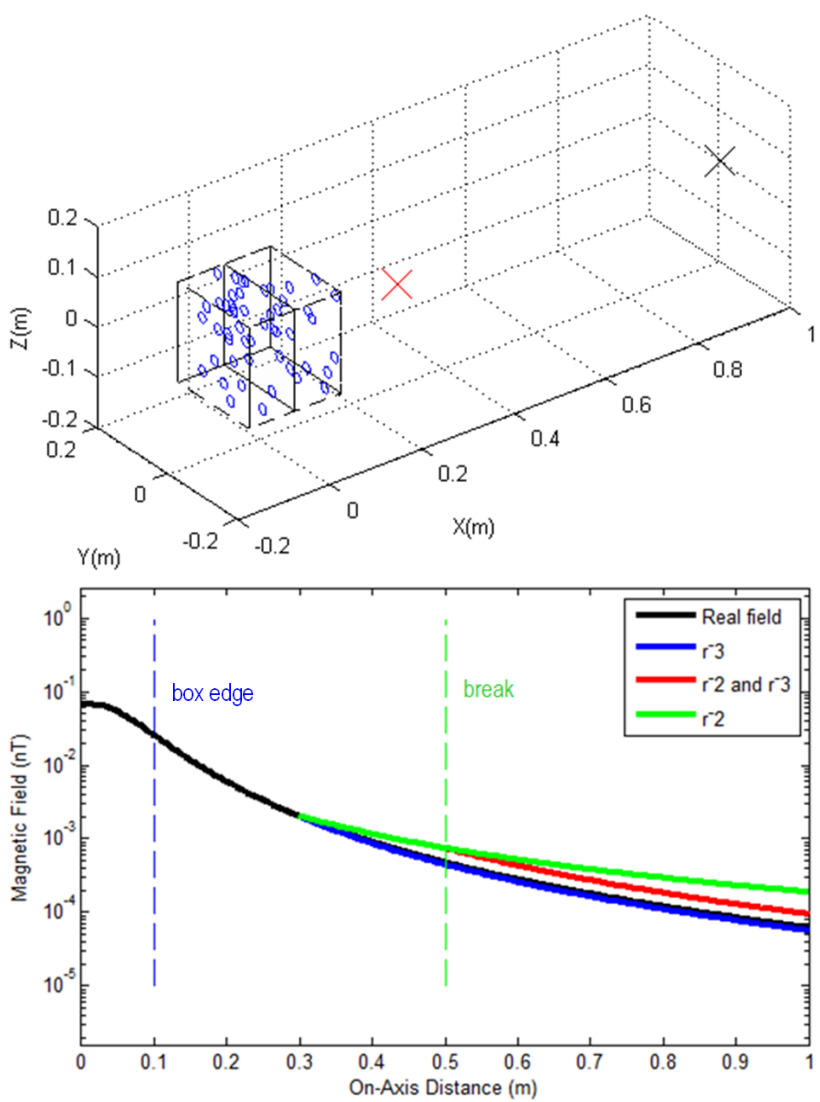

Fig. 3. (a) Model orientation set-up for multiple small dipoles. The red cross indicates the measurement location and the black cross indicates the extrapolation location. (b) Modelling the field fall-off from multiple small dipoles with random positions.

assuming a source origin at the centre of the box (i.e. the magnetic-field falls-off according to the power law with respect to the box centre).

5. Different extrapolation techniques are compared by calculating their deviation from the true magnetic-field strength at the extrapolation distance. We define $R$ to be the ratio of the extrapolated value to the true value (i.e. $R=2$ indicates the magnitude of the extrapolated field is twice the real value, while for $R=0.5$ the extrapolated field is half the real value).

6. If the test uses multiple current loops, the process is repeated for a variation in the current loop positions as defined in step 2.

\section{Model demonstration}

Figure 2 shows the model set-up and the magnetic-field falloff by a single current loop in the centre of a cubic box of width $0.20 \mathrm{~m}$. Additional model parameters are displayed in Table 1 . In this ideal scenario the field falls off very close to
Table 2. Multiple dipole model parameters.

\begin{tabular}{ll}
\hline Parameter & \\
\hline Loop radius & $0.01 \mathrm{~m}$ \\
Loop current & $1 \mathrm{~mA}$ \\
Loop position & Random \\
Loop orientation & on-axis $x$ axis \\
Number of loops & 50 \\
Box width & $0.20 \mathrm{~m}$ \\
Verification distance & $0.30 \mathrm{~m}$ \\
Break distance & $0.50 \mathrm{~m}$ \\
Extrapolation distance & $1.00 \mathrm{~m}$ \\
\hline
\end{tabular}

the dipole approximation, with the more cautious techniques overestimating the field.

Having one large current loop is, however, unrepresentative of a real piece of equipment. In reality most units are composed of small electrical components each with their own corresponding small current loop. An electronics board is a good example of this. In order to model this scenario, a large number of small current loops were placed in a random position inside the box. This allows for the fact that electronics boards are not restricted to the centre plane of a unit. All of the loops were aligned along the $x$ axis to produce a worst-case scenario. The resulting field fall-off is similar to the single large current loop case, but provided a different set of $R$ ratios for each new set of random positions, according to step 6 of the procedure. The resulting field fall-off can be observed in Fig. 3 for the parameters given in Table 2.

We now investigate the impact of varying the verification and break distances. We chose to vary the verification distance between $0.20-0.45 \mathrm{~m}$ in $0.10 \mathrm{~m}$ steps (with a fixed break distance of $0.50 \mathrm{~m}$ ). We also chose to vary the break distance between $0.20-1.00 \mathrm{~m}$ in $0.10 \mathrm{~m}$ steps (with a fixed verification distance at $0.20 \mathrm{~m}$ ). We observe how the ratio $R$ varies for the different test cases.

\section{Results}

We display the information in the form of a box and whisker plot, described pictorially in Fig. 4. For a chosen verification distance and extrapolation distance, 50 random multiple dipole positions were chosen to build up a statistical picture of the field fall-off in different cases. The choice of parameters for the two cases studied are shown in Tables 3 and 4 .

Firstly, we find that as the verification distance increases, the precision of the extrapolated field improves (Fig. 5), because the further a measurement is taken from the box edge, the more valid the centre dipole approximation becomes. We notice that the dipole approximation with a scaling power of -3 is the most accurate - with an average field prediction closest to the real value. However, in approximately $50 \%$ of tested cases this results in an underestimation of the field 


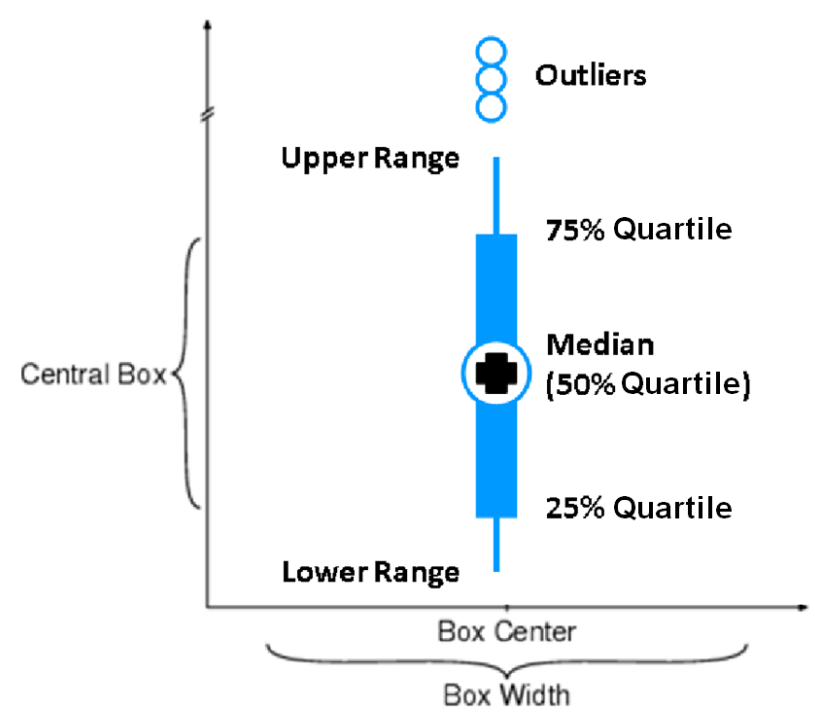

Fig. 4. Box and whisker definition as used in this study.

Table 3. Parameter selection for the investigation of varying verification distance.

\begin{tabular}{ll}
\hline Parameter & \\
\hline Loop radius & $0.01 \mathrm{~m}$ \\
Loop current & $1 \mathrm{~mA}$ \\
Loop position & Random \\
Loop orientation & on-axis $x$ axis \\
Number of loops & 50 \\
Box width & $0.10 \mathrm{~m}$ \\
Verification distance & Varies from $0.10 \mathrm{~m}$ to $0.45 \mathrm{~m}$ \\
Break distance & $0.50 \mathrm{~m}$ \\
Extrapolation distance & $1.00 \mathrm{~m}$ \\
Number of independent trials & 50 \\
\hline
\end{tabular}

magnitude at the extrapolation distance. This introduces risk, since it is possible the EUT emissions may be deemed acceptable through analysis yet fail in practice. Using a scaling power of -2 was found to never underestimate the real value, while the combined extrapolation technique underestimated the real value in $20-40 \%$ of tested cases.

There are outliers that generate very large overestimations in the prediction (very large values of $R$ ). These cases emerge when the random selection of loop position places the majority of loops around the box edges. This deviates from our extrapolation assumption that the EUT source emits from the centre of its volume, yielding a large value for $R$. This fact does not invalidate the method, however, since we do not assume any knowledge of the EUT wiring geometry before performing the extrapolation.

We now study the effect of varying the break distance for the parameters shown in Table 4. As before, the dipole scaling power of -3 under predicts the field in approximately
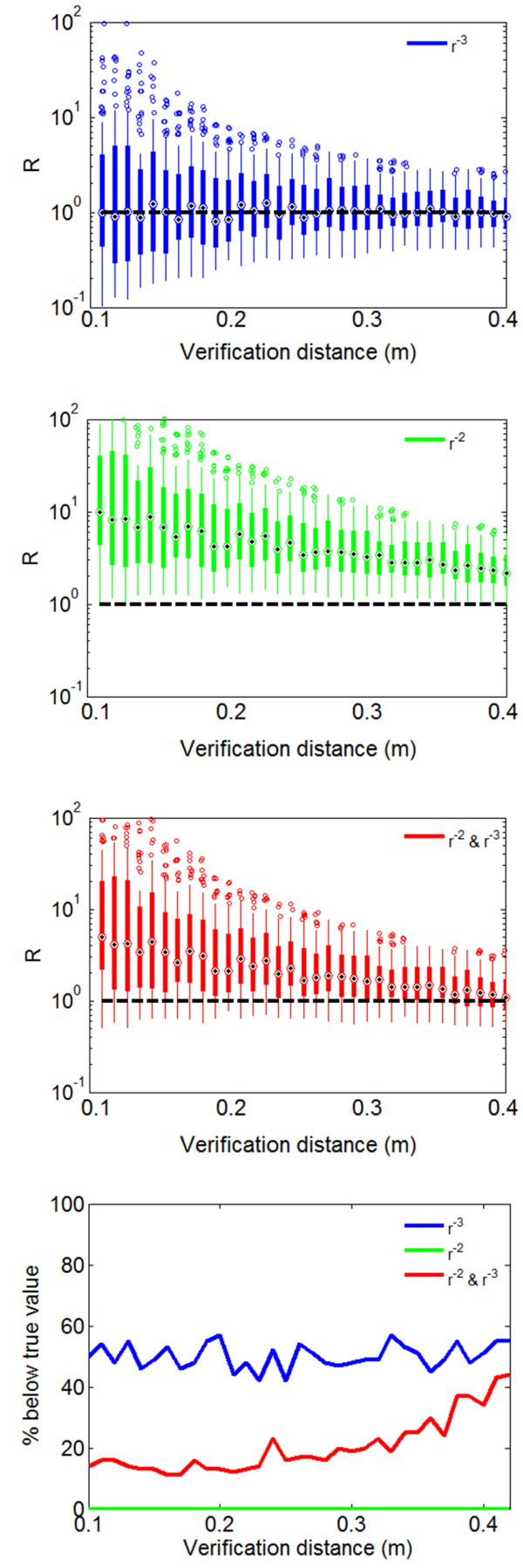

Fig. 5. Ratio of extrapolated to actual field, $R$, for the on-axis magnetic field at the extrapolation distance of $1.0 \mathrm{~m}$, for different scaling powers. The dipole scaling power of -3 is shown in blue, a scaling power of -2 is shown in green, and a scaling power of -2 followed by -3 after the break point is shown in red. The percentage of trials that resulted in an underestimation of the field are displayed for the three extrapolation techniques. The break point is held constant at $0.5 \mathrm{~m}$, while the verification distance is varied from the box edge out to $0.45 \mathrm{~m}$. The statistical results for each verification distance represent 50 independent trials, each of which uses the configuration summarised in Table 3. 

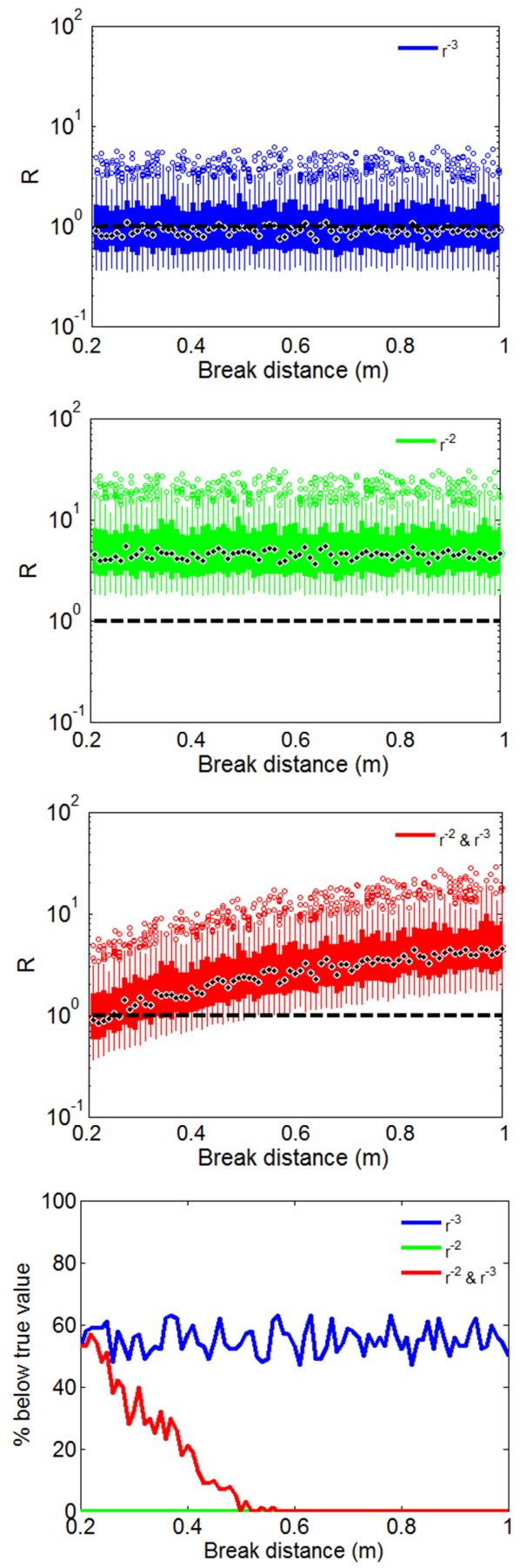

Fig. 6. As in Fig. 5, except now the verification distance is held constant at $0.20 \mathrm{~m}$, while the verification distance is varied from the verification distance to the extrapolation distance at $1.00 \mathrm{~m}$, for the configuration summarised in Table 4.
Table 4. Parameter selection for the investigation of varying break distance.

\begin{tabular}{ll}
\hline Parameter & \\
\hline Loop radius & $0.01 \mathrm{~m}$ \\
Loop current & $1 \mathrm{~mA}$ \\
Loop position & Random \\
Loop orientation & on-axis $x$ axis \\
Number of loops & 50 \\
Box width & $0.20 \mathrm{~m}$ \\
Verification distance & $0.20 \mathrm{~m}$ \\
Break distance & Varies from $0.20 \mathrm{~m}$ to $1.00 \mathrm{~m}$ \\
Extrapolation distance & $1.00 \mathrm{~m}$ \\
Number of independent trials & 50
\end{tabular}

$50 \%$ of tested cases (Fig. 6). The suggested scaling power of -2 is never found to under predict the field; however, it is found to commonly over predict by a factor of 5 . By switching the scaling power from -2 to -3 after the break distance, the resulting prediction is more accurate than a fixed scaling power of -2 . We find that the extrapolation reliably avoids under prediction when a distance of 3 times the unit size is selected (in the case above a distance of $0.60 \mathrm{~m}$ ), deduced from the distance at which the red line in Fig. 6 reaches zero. We find an average over prediction by a factor of 2, equivalent to a 2.5 times accuracy improvement over using a fixed scaling power of -2 .

In previous literature empirical measurements suggest it is considered safe to use the dipole approximation at a distance equal to a factor of 5 times the unit size (Junge and Marliani, 2011). In these traditional cases measurements were taken further that this safe distance, therefore no prior extrapolation was needed. This is not the same in our method, however, because of the cautious use of a scaling power of -2 before the break distance, which gives a reliable overestimation up to that point. Thus, a break distance of 3 times the unit size can be implemented, even though it is smaller than the factor of 5 times the unit size judged acceptable for dipolar use.

\section{Conclusions}

AC magnetic-field verification of individual spacecraft units is becoming increasingly challenging, requiring measurements to be taken close to the equipment under test (EUT) and extrapolated. We find that using a traditional extrapolation technique that use a dipolar scaling power of -3 close to the EUT risks underestimating the field emission. To avoid this, we propose an extrapolation that uses a magnetic-field scaling power of -2 up to a break distance, after which a scaling power of -3 is used. We find the optimum break distance to be 3 times the unit size. This method avoids underestimating the field in the test cases modelled, while demonstrating an accuracy 2.5 times better than a method that uses 
a fixed scaling power of -2 alone. This demonstrates the avoidance of a severe over prediction or under prediction of the magnetic-field emission.

It is recommended that for each unit that cannot meet the verification requirements using traditional measurement techniques, the extrapolation method described should be implemented. It is recognised that the improvement in accuracy using this new method will differ for each spacecraft unit, since each will have a unique geometrical size and separation from the magnetometer. A specific comparison between the new method and baseline proposals for extrapolation can be made for each unit to be placed on the spacecraft, estimating an individual extrapolation accuracy for each unit.

\section{Further work}

If the frequency sources can be identified in advance, the use of a multiple dipole analysis technique similar to that used on DC magnetic fields could be used to more accurately model emissions from the EUT, which can then be extrapolated to obtain a significant accuracy improvement.

Equally, the use of a gradiometer technique could be used to greatly improve the extrapolation accuracy by increasing the number of measurement points and therefore the amount of information available to provide a magnetic-field model fit.

Acknowledgements. M. A. Pudney is grateful for the financial support from the STFC and Astrium Ltd.

Edited by: L. Vazquez

\section{References}

Filippopoulos, G. and Tsanakas, D.: Analytical calculation of the magnetic field produced by electric power lines, IEEE T. Power Deliver., 20, 1474-1482, 2005.

Jackson, J. D.: Classical Electrodynamics, New York, John Wiley and Sons, 1999.

Junge, A. and Marliani, F.: Prediction of DC Magnetic Fields for Magnetic Cleanliness on Spacecraft 2011, IEEE International Symposium on Electromagnetic Compatibility (EMC), Rome, 834-839, 2011.

Müller, D., Marsden, R. G., St. Cyr, O. C., and Gilbert, H. R.: Solar Orbiter. Exploring the Sun-Heliosphere Connection, Sol. Phys., 285, 25-70, 2013.

National Grid: How the field from a power line falls with distance, Retrieved 9 October 2012, http://www.emfs.info/Sources+of+ EMFs/Overhead+power+lines/power+line+power+law.htm (last access: 25 July 2013), 2012. 